\title{
Efficacy and safety of tigecycline monotherapy vs. imipenem/cilastatin in Chinese patients with complicated intra-abdominal infections: a randomized controlled trial
}

Zhangjing Chen ${ }^{1}$, Jufang $\mathrm{Wu}^{1}$, Yingyuan Zhang ${ }^{1 *}$, Junming $\mathrm{Wei}^{2}$, Xisheng Leng ${ }^{3}$, Jianwei $\mathrm{Bi}^{4}$, Rong Li $\mathrm{i}^{5}$, Lunan Yan $^{6}$,

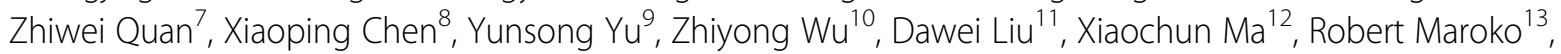
Angel Cooper ${ }^{13}$

\begin{abstract}
Background: Tigecycline, a first-in-class broad-spectrum glycylcycline antibiotic, has broad-spectrum in vitro activity against bacteria commonly encountered in complicated intra-abdominal infections (c|Als), including aerobic and facultative Gram-positive and Gram-negative bacteria and anaerobic bacteria. In the current trial, tigecycline was evaluated for safety and efficacy vs. imipenem/cilastatin in hospitalized Chinese patients with clAls.

Methods: In this phase 3, multicenter, open-label study, patients were randomly assigned to receive IV tigecycline or imipenem/cilastatin for $\leq 2$ weeks. The primary efficacy endpoints were clinical response at the test-of-cure visit (12-37 days after therapy) for the microbiologic modified intent-to-treat and microbiologically evaluable populations. Because the study was not powered to demonstrate non-inferiority between tigecycline and imipenem/cilastatin, no formal statistical analysis was performed. Two-sided 95\% confidence intervals (Cls) were calculated for the response rates in each treatment group and for differences between treatment groups for descriptive purposes.
\end{abstract}

Results: One hundred ninety-nine patients received $\geq 1$ dose of study drug and comprised the modified intent-totreat population. In the microbiologically evaluable population, $86.5 \%$ (45 of 52) of tigecycline- and $97.9 \%$ (47 of 48) of imipenem/cilastatin-treated patients were cured at the test-of-cure assessment (12-37 days after therapy); in the microbiologic modified intent-to-treat population, cure rates were $81.7 \%$ (49 of 60) and $90.9 \%$ (50 of 55), respectively. The overall incidence of treatment-emergent adverse events was $80.4 \%$ for tigecycline vs. $53.9 \%$ after imipenem/cilastatin therapy $(P<0.001)$, primarily due to gastrointestinal-related events, especially nausea $(21.6 \%$ vs. $3.9 \% ; P<0.001)$ and vomiting $(12.4 \%$ vs. $2.0 \% ; P=0.005)$.

Conclusions: Clinical cure rates for tigecycline were consistent with those found in global CIAl studies. The overall safety profile was also consistent with that observed in global studies of tigecycline for treatment of clAl, as well as that observed in analyses of Chinese patients in those studies; no novel trends were observed.

Trial Registration: ClinicalTrials.gov NCT00136201

\footnotetext{
* Correspondence: yyzhang39@hotmail.com

${ }^{1}$ Huashan Hospital, Fudan University, Shanghai, China
} 


\section{Background}

The management of complicated intra-abdominal infections (cIAIs) remains a challenge to physicians because of their polymicrobial nature coupled with the high risk of sequelae and mortality in severely ill patients with these infections [1-3]. While most infections contain a mixture of aerobic and anaerobic bacteria with a preponderance of Enterobacteriaceae (e.g., Escherichia coli) $[1,2]$, resistant and uncommon organisms (e.g., Enterococcus, Staphylococcus, Enterobacter, Pseudomonas and Candida spp.) are often isolated in patients with nosocomial infection or tertiary peritonitis [4].

Selection of empiric antimicrobial therapy must consider the likelihood of encountering isolates that possess multiple resistance factors (e.g., extended-spectrum betalactamases [ESBLs], vancomycin-resistant enterococci [VRE]) $[1,2]$. Recently published treatment guidelines recommend broad-spectrum monotherapy or combination regimens (e.g., carbapenem monotherapy, third- or fourth-generation cephalosporins or fluoroquinolones plus metronidazole) for high-risk patients with severe or postoperative nosocomial intra-abdominal infections wherein polymicrobial infections and/or resistant flora are more prevalent $[1,2]$. Notably, inappropriate antibiotic choices have been linked to delayed clinical resolution, longer hospital stay, and an increased risk of mortality [5,6]. While adjunctive antimicrobial therapy is vital to achieving desired outcomes, surgical intervention is essential in the management of patients with cIAIs.

Tigecycline, a first-in-class expanded broad-spectrum glycylcycline antibiotic approved for use in patients with cIAIs, overcomes the 2 major mechanisms of resistance to tetracycline (i.e., drug-specific efflux pump acquisition and ribosomal protection) $[7,8]$. Tigecycline has broadspectrum in vitro activity against bacteria commonly encountered in cIAIs, including aerobic and facultative Gram-positive and Gram-negative bacteria and anaerobic bacteria [9-11]. Furthermore, tigecycline has in vitro activity against multidrug-resistant bacteria such as VRE, ESBL- and carbapenemase-producing enteric Gram-negative bacteria, and methicillin-resistant S. aureus (MRSA) [12-14]. Tigecycline also exhibits linear pharmacokinetics and has a large volume of distribution, suggesting extensive tissue penetration [15].

Two global phase 3 double-blind trials, which compared the efficacy of tigecycline and imipenem/cilastatin in hospitalized patients with cIAIs, have demonstrated that tigecycline is efficacious for this condition [16]. Imipenem/cilastatin was chosen as the comparative agent because it has a wide spectrum of activity, it is effective in the treatment of hospitalized patients with intraabdominal infections, and is widely available and used in the treatment of cIAI. In the current trial, tigecycline monotherapy was evaluated for safety and efficacy vs. imipenem/cilastatin in hospitalized Chinese patients with cIAI as a supplement to the 2 double-blinded pivotal global studies in cIAI [16].

\section{Methods}

\section{Study design and treatment regimens}

This study was a phase 3, multicenter, open-label trial of hospitalized Chinese patients at least 18 years of age who were candidates for or had undergone a laparotomy, laparoscopy, or percutaneous drainage of an intraabdominal abscess and had a known or suspected diagnosis of cIAI. Specific enrollment criteria are outlined in Table 1. Following approval of the study protocol by the institutional review board or ethical review committee at each participating center, each patient or his or her legal representative provided written informed consent prior to undergoing any study procedures.

Patients were randomly assigned using a computerized enrollment system in a $1: 1$ ratio to receive tigecycline (initial 100-mg dose given by intravenous [IV] infusion over a 30-minute period, followed by $50 \mathrm{mg}$ IV every 12 hours) or IV imipenem/cilastatin $(500 \mathrm{mg} / 500 \mathrm{mg}$ every 6 hours or dose-adjusted based on weight and creatinine clearance). Patients were to receive study drug for up to 2 weeks, unless deemed a treatment failure after at least 4 doses of tigecycline or 8 doses of imipenem/cilastatin.

Baseline aerobic and anaerobic cultures from the primary intra-abdominal site of infection and two sets of blood cultures were obtained within 24 hours of the first dose of study drug.

\section{Clinical and microbiologic evaluations}

At serial visits throughout the study, the clinical status of the patient's intra-abdominal infection was assessed based upon the presence or absence of the following signs and symptoms: fever; localized or diffuse abdominal wall rigidity or involuntary guarding; abdominal tenderness or pain; ileus or hypoactive bowel sounds; nausea or vomiting. The clinical response to study drug was determined by the investigator at the test-of-cure (TOC) visit (12-37 days after therapy) and categorized as cure, failure, or indeterminate.

Microbiologic response by patient was categorized at the TOC visit as eradication, persistence, superinfection (i.e., the emergence of a new isolate was documented at the site of infection with worsening signs and symptoms of infection), or indeterminate. The microbiologic response for each baseline isolate at the TOC visit was categorized as eradication, persistence, or indeterminate.

\section{Safety and tolerability assessments}

All patients who received at least one dose of study drug were evaluated for safety. Safety was assessed from clinical observations and findings from serial electrocardiograms 
Table 1 Enrollment criteria

\begin{tabular}{|c|c|}
\hline Inclusion* & Exclusion $^{\dagger}$ \\
\hline $\begin{array}{l}\text { Men and non-pregnant, non-lactating women } \geq 18 \text { years of age who } \\
\text { required a surgical procedure for a complicated intra-abdominal infection } \\
\text { (clAl) } \\
\text { ClAl defined as the following: } \\
\text { An intra-abdominal abscess (including liver and spleen) that developed in } \\
\text { a postsurgical patient after receiving standard antibacterial therapy (i.e., at } \\
\text { least } 48 \text { hours, but not more than } 5 \text { days of antibiotics); } \\
\text { Appendicitis complicated by perforation and/or a periappendiceal } \\
\text { abscess; } \\
\text { Perforated diverticulitis complicated by abscess formation or fecal } \\
\text { contamination; } \\
\text { Complicated cholecystitis with evidence of perforation or empyema; } \\
\text { perforation of a gastric or duodenal ulcer with symptoms exceeding } 24 \\
\text { hours; } \\
\text { Purulent peritonitis or peritonitis associated with fecal contamination; } \\
\text { Perforation of the large or small intestine with abscess or fecal } \\
\text { contamination, or traumatic bowel perforation with symptoms lasting at } \\
\text { least } 12 \text { hours before an operation }\end{array}$ & $\begin{array}{l}\text { Preoperative suspicion of a diagnosis of spontaneous bacterial peritonitis, } \\
\text { simple cholecystitis, gangrenous cholecystitis without rupture, simple } \\
\text { appendicitis, acute suppurative cholangitis, pancreatic abscess, or } \\
\text { infected necrotizing pancreatitis; } \\
\text { Acute Physiologic and Chronic Health Evaluation (APACHE) II score } \\
\text { greater than 30; } \\
\text { Surgical procedure requiring that fascia or deep muscular layers be left } \\
\text { open or expectation of planned abdominal re-exploration either in or } \\
\text { out of the operating room; } \\
\text { Use of immunosuppressive therapy that would decrease the patient's } \\
\text { ability to eradicate the infection, including use of high-dose } \\
\text { corticosteroids (e.g., } 40 \text { mg or more of prednisone or an equivalent per } \\
\text { day for more than } 3 \text { weeks before randomization) or known diagnosis of } \\
\text { acquired immunodeficiency syndrome; } \\
\text { Current intra-abdominal infection known to be caused by one or more } \\
\text { bacterial isolates not susceptible to either of the study drugs (e.g., } P \text {. } \\
\text { aeruginosa, Proteus mirabilis); } \\
\text { Active or treated leukemia or systemic malignancy that requires } \\
\text { chemotherapy, immunotherapy, radiation therapy, or antineoplastic } \\
\text { therapy within the } 3 \text { months before enrollment, or any metastatic } \\
\text { malignancy to the abdomen with life expectancy < } 6 \text { months; } \\
\text { Presence of any uncontrolled central nervous system disease; } \\
\text { Significant hepatic disease (i.e., aspartate aminotransferase [AST] or } \\
\text { alanine aminotransferase [ALT] level }>10 \text { times the upper limit of normal } \\
\text { [ULN] or total bilirubin value }>3 \text { times the ULN) or acute hepatic failure } \\
\text { or acute decompensation of chronic hepatic failure; } \\
\text { Significant renal disease (i.e., calculated creatinine clearance }<41 \text { mL/ } \\
\text { min/1.73 m² after adequate hydration); } \\
\text { Neutropenia with absolute neutrophil count < } 1000 \text { mm } 3 \text { (however, } \\
\text { neutrophil counts as low as } 500 \text { cells/mm } 3 \text { permitted if secondary to the } \\
\text { acute infectious process); } \\
\text { Concomitant treatment with ganciclovir }\end{array}$ \\
\hline
\end{tabular}

*Patients had to satisfy the inclusion criteria to be considered eligible for study participation

${ }^{\dagger}$ Patients were ineligible for study participation if they had one or more of the listed exclusions

(ECGs), serum chemistry, hematology, coagulation, and urinalysis tests. Adverse events (AEs) were recorded throughout the study period, up to and including the TOC visit or 14 days after last dose of study medication (whichever was longer), and were subjectively rated by the investigator as to their severity and relationship to the study drug. Investigators also recorded whether the AE resulted in temporary or permanent discontinuation and whether any remedial action was taken. Serious AEs (SAEs; i.e., those that were life-threatening, led to prolongation of the existing hospitalization, caused persistent or significant disability or incapacity, led to cancer, or death) were also recorded.

\section{Statistical analysis}

Three primary populations of patients were assessed for safety, clinical, and bacteriologic outcomes; the modified intent-to-treat (mITT), microbiologic modified ITT (mmITT), and the microbiologically evaluable (ME) populations. ITT patients who received at least one dose of study drug were included in the mITT population. Patients in the mITT population who had clinical evidence of complicated intra-abdominal infection comprised the clinical-mITT (c-mITT) population. The
m-mITT population consisted of patients in the c-mITT population who had $\geq 1$ isolate identified at the baseline assessment. Clinically evaluable (CE) patients were cmITT patients who met all inclusion/exclusion criteria; did not receive concomitant antibiotics after the baseline intra-abdominal culture was obtained through the testof-cure visit; received no more than 1 dose of a prohibited antibacterial treatment after the baseline intraabdominal culture was obtained but before the first dose of study drug; received at least 5 days of study drug and between $80 \%$ and $120 \%$ of planned doses; had a test-ofcure visit 12 to 37 days after the last dose of study drug. Those patients included in the microbiologically evaluable (ME) population were CE patients who had at least one identifiable baseline bacterial isolate(s) taken from the primary site of infection that was susceptible to both study drugs and who had a microbiologic response assigned, i.e., eradication, persistence, or superinfection, at the TOC visit.

This trial was designed to enroll 200 patients. By assuming an evaluability rate of $50 \%$, this would allow for the evaluation of $100 \mathrm{ME}$ patients. The expected percentage of patients with a favorable clinical response (cure) was $70 \%$ at the TOC assessment. With a sample 
size of 50 evaluable patients in a treatment group, if 35 patients showed a favorable clinical response, then the 2-sided exact 95\% CI would equal 55.4, 82.1.

The primary efficacy endpoints were clinical response at the TOC visit for the $\mathrm{m}$-mITT and ME populations. Microbiological response at the TOC visit by patient and isolate was performed as a secondary analysis. Because the study was not powered to demonstrate non-inferiority between tigecycline and imipenem/cilastatin, no formal statistical analysis was performed for the primary and secondary efficacy endpoints of this study. Two-sided $95 \%$ confidence intervals (CIs) were calculated for the response rates in each treatment group for descriptive purposes using the "exact" method of Clopper and Pearson. Two-sided 95\% CIs for differences between groups were calculated based on the asymptotic method corrected for continuity, except for differences in subgroup analyses where the Wilson score method corrected for continuity was used. Secondary efficacy analyses included the determination of susceptibility to tigecycline $\left(\mathrm{MIC}_{50}\right.$, $\left.\mathrm{MIC}_{90}\right)$ and the development of decreased susceptibility (at least a 4-fold increase in MIC from baseline). Susceptibility was analyzed by using the Fisher exact test.

A post-hoc Cochran-Mantel-Haenszel analysis (stratified by protocol) was performed on the 2 co-primary efficacy endpoints to evaluate equality across the current trial and the 2 global cIAI studies [16]. The Breslow-Day test was used to evaluate equality across the strata with a $P$ value of $<0.05$ indicating statistical significance from study to study with respect to clinical response.

The Global Biostatistics and Programming Department of Wyeth Research performed all statistical analyses.

\section{Results}

The disposition of Chinese patients participating in this trial and the analysis populations are summarized in Figure 1. Overall, 199 patients received at least 1 dose of a study drug and comprised the mITT (safety) population.

The demographic and baseline medical characteristics for the mITT patients were comparable between the 2 treatment groups (Table 2) with the exception that tigecycline-treated patients were statistically significantly older $(P=0.021)$ and the severity of intra-abdominal illness was statistically significantly greater in the tigecycline cohort (mean APACHE II score was 5.1 for tigecycline vs. 4.1 for imipenem/cilastatin; $P=0.038$ ). Complicated appendicitis was the most common intraabdominal infection diagnosis in both groups $(76.3 \%$ vs. $76.5 \%$ for tigecycline and imipenem/cilastatin, respectively).

\section{Clinical efficacy}

For the ME population, clinical cure rates were $86.5 \%$ for tigecycline and $97.9 \%$ for imipenem/cilastatin (95\% CI for the difference, -23.5, 0.7) (Table 3). All patients had APACHE II scores $\leq 15$. Corresponding clinical cure rates for the m-mITT population were $81.7 \%$ and $90.9 \%$, respectively (95\% CI for the difference, $-23.4,4.9)$. Clinical cure rates stratified by monomicrobial and polymicrobial infections are found in Table 3. For complicated appendicitis, by far the most frequent diagnosis in this study, clinical cure rates at the TOC visit for the ME population were $87.0 \%$ for tigecycline and $100.0 \%$ for imipenem/cilastatin (95\% CI for the difference, -27.0 , -0.6) (Table 4).

These findings in Chinese patients were similar to results in 2 global double-blind clinical trials [16], wherein the clinical response of tigecycline was found to be non-inferior to imipenem/cilastatin (Figure 2). The results of the Breslow-Day test indicate that there was no significant difference in clinical response across the 3 studies in the ME $(P=0.0979)$ or the m-mITT population $(P=0.1655)$ for the current study in Chinese patients (Study 316) and the 2 pivotal global studies (Study 301 and Study 306).

A total of 6 tigecycline- and 3 imipenem/cilastatintreated patients in the ME population had positive pretherapy blood culture results, including 7 isolates in tigecycline patients and 4 in imipenem/cilastatin patients. All blood culture isolates were Gram-negative rods. All 9 patients with bacteremia were reported as having a clinical cure/bacteriologic eradication at the TOC visit.

\section{Microbiologic efficacy}

For the ME population, presumed eradication of intraabdominal isolates at the patient level based upon clinical response mirrored the clinical cure rates: $86.5 \%$ for tigecycline- and $97.9 \%$ for imipenem/cilastatin-treated patients (Table 5). Eradication rates stratified by monomicrobial and polymicrobial infection are also summarized in Table 5.

Eradication rates at the TOC visit for the most common types of isolated intra-abdominal pathogens are outlined in Table 6 for the two treatment groups. For $E$. coli, the most commonly isolated bacteria, eradication rates were $88.1 \%$ for tigecycline vs. $97.7 \%$ for imipenem/ cilastatin. There were no obvious differences in eradication rates of other aerobic and anaerobic bacteria, although the number of isolates per species was small, making comparisons difficult.

Overall, MIC values for tigecycline against the most commonly isolated aerobes and anaerobes was $\leq 2.0 \mu \mathrm{g} /$ $\mathrm{mL}$. For E. coli $(\mathrm{n}=86), \mathrm{MIC}_{50}$ and $\mathrm{MIC}_{90}$ values were $0.125 \mu \mathrm{g} / \mathrm{mL}$ and $0.5 \mu \mathrm{g} / \mathrm{mL}$ for tigecycline, respectively, 




Figure 1 Analysis populations and patient disposition: Tigecycline (TGC) vs. imipenem-cilastatin (I/C) in complicated intra-abdominal infections.

and $0.25 \mu \mathrm{g} / \mathrm{mL}$ and $0.5 \mu \mathrm{g} / \mathrm{mL}$ for imipenem/cilastatin, respectively. For patients in the tigecycline group who had persistent $E$. coli infections (5 isolates; $11.9 \%$ ), MIC values ranged from 0.125 to $0.5 \mu \mathrm{g} / \mathrm{mL}$. Bacterial susceptibilities to tigecycline were consistent with clinical responses, and no isolates from later cultures with a decreased susceptibility ( $\geq 4$-fold increase in MIC from baseline) to tigecycline were identified.

\section{Safety and tolerability}

The mITT population received a median of 5 and 6 days of tigecycline or imipenem/cilastatin treatment, 
Table 2 Demographic and baseline characteristics (mITT population)

\begin{tabular}{|c|c|c|c|}
\hline & Tigecycline $\mathrm{N}=97$ & Imipenem/cilastatin $\mathrm{N}=102$ & $P$ value* \\
\hline Mean \pm SD age, years & $46.8 \pm 18.2$ & $41.0 \pm 16.7$ & 0.021 \\
\hline Sex, $n(\%)$ male & $65(67.0)$ & $71(69.6)$ & 0.694 \\
\hline Mean \pm SD weight, $\mathbf{k g}$ & $63.2 \pm 10.9$ & $64.7 \pm 11.1$ & 0.335 \\
\hline Mean \pm SD creatinine clearance, $\mathrm{mL} / \mathrm{min}$ & $100.9 \pm 37.5$ & $108.8 \pm 37.1$ & 0.138 \\
\hline APACHE II score & & & 0.038 \\
\hline Mean \pm SD & $5.1 \pm 3.9$ & $4.1 \pm 2.7$ & \\
\hline Median (range) & $4.0(0.0-21.0)$ & $4.0(0.0-12.0)$ & \\
\hline Primary intra-abdominal diagnosis, $\mathrm{n}(\%)$ & & & 0.509 \\
\hline Complicated appendicitis & $74(76.3)$ & $78(76.5)$ & \\
\hline Complicated cholecystitis & $5(5.2)$ & $7(6.9)$ & \\
\hline Perforated duodenal ulcer & $3(3.1)$ & $7(6.9)$ & \\
\hline Peritonitis due to perforation of small intestine & $6(6.2)$ & $2(2.0)$ & \\
\hline Peritonitis due to perforation of large intestine & $4(4.1)$ & $2(2.0)$ & \\
\hline Perforated gastric ulcer & $1(1.0)$ & $2(2.0)$ & \\
\hline Complicated cholangitis & $2(2.1)$ & $0(0)$ & \\
\hline Post-traumatic peritonitis & $1(1.0)$ & $1(1.0)$ & \\
\hline Liver abscess & $1(1.0)$ & $2(2.0)$ & \\
\hline Perforated stomach & $0(0)$ & $1(1.0)$ & \\
\hline
\end{tabular}

$\mathrm{NA}=$ not available

*Categorical baseline demographic and medical variables were analyzed using the Fisher exact test. Continuous variables were compared using a one-way analysis of variance (ANOVA) model with treatment as a factor.

respectively. The overall range for the number of days of therapy was 2 to 14 days. The overall incidence of treatment-emergent AEs was $80.4 \%$ for tigecycline vs. $53.9 \%$ for imipenem/cilastatin therapy $(P<0.001)$; this difference between treatment groups was primarily due to gastrointestinal-related events, primarily nausea and vomiting (Table 7 ). The overall percentage of subjects with study drug-related AEs was significantly higher in the tigecycline group compared to the imipenem/cilastatin treatment arm $(55.7 \%$ vs. $41.2 \% ; P<0.05)$. The difference stemmed primarily from between-group differences in nausea (20.6\% tigecycline vs. $2 \%$ imipenem/cilastatin; $P<0.001$ ) and vomiting (tigecycline $10.3 \%$ vs. imipenem/cilastatin $1 \% ; P=0.004)$. Most AEs

Table 3 Clinical cure rates* by analysis population at test-of-cure visit

\begin{tabular}{|c|c|c|c|c|c|}
\hline \multirow[b]{2}{*}{ Population } & \multicolumn{2}{|c|}{ Tigecycline } & \multicolumn{2}{|c|}{ Imipenem/cilastatin } & \multirow{2}{*}{$\begin{array}{c}\text { Difference } \\
\text { (Tigecycline-Imipenem/cilastatin) }\end{array}$} \\
\hline & $\mathrm{N}$ & $\%(95 \% \mathrm{Cl})$ & $\mathrm{N}$ & $\%(95 \% \mathrm{Cl})$ & \\
\hline$\overline{\mathrm{ME}}$ & $45 / 52$ & $\begin{array}{c}86.5 \\
(74.2,94.4)\end{array}$ & $47 / 48$ & $\begin{array}{c}97.9 \\
(88.9,99.9)\end{array}$ & $\begin{array}{c}-11.4 \\
(-23.5,0.7)\end{array}$ \\
\hline Monomicrobial & $30 / 33$ & $\begin{array}{c}90.9 \\
(75.7,98.1)\end{array}$ & $25 / 26$ & $\begin{array}{c}96.2 \\
(80.4,99.9)\end{array}$ & $\begin{array}{c}-5.2 \\
(-22.0,13.7)\end{array}$ \\
\hline Polymicrobial & $15 / 19$ & $\begin{array}{c}78.9 \\
(54.4,93.9)\end{array}$ & $22 / 22$ & $\begin{array}{c}100.0 \\
(84.6,100.0)\end{array}$ & $\begin{array}{c}-21.1 \\
(-46.1,2.2)\end{array}$ \\
\hline $\mathrm{m}-\mathrm{mlTT}$ & $49 / 60$ & $\begin{array}{c}81.7 \\
(69.6,90.5)\end{array}$ & $50 / 55$ & $\begin{array}{c}90.9 \\
(80.0,97.0)\end{array}$ & $\begin{array}{c}-9.2 \\
(-23.4,4.9)\end{array}$ \\
\hline Monomicrobial & $32 / 38$ & $\begin{array}{c}84.2 \\
(68.7,94.0)\end{array}$ & $27 / 29$ & $\begin{array}{c}93.1 \\
(77.2,99.2)\end{array}$ & $\begin{array}{c}-8.9 \\
(-26.0,10.7)\end{array}$ \\
\hline Polymicrobial & $17 / 22$ & $\begin{array}{c}77.3 \\
(54.6,92.2)\end{array}$ & $23 / 26$ & $\begin{array}{c}88.5 \\
(69.8,97.6)\end{array}$ & $\begin{array}{c}-11.2 \\
(-35.8,13.0)\end{array}$ \\
\hline CE & $67 / 77$ & $\begin{array}{c}87.0 \\
(77.4,93.6)\end{array}$ & $83 / 87$ & $\begin{array}{c}95.4 \\
(88.6,98.7)\end{array}$ & $\begin{array}{c}-8.4 \\
(-18.3,1.5)\end{array}$ \\
\hline$c-m l T T$ & $78 / 97$ & $\begin{array}{c}80.4 \\
(71.1,87.8)\end{array}$ & $88 / 98$ & $\begin{array}{c}89.8 \\
(82.0,95.0)\end{array}$ & $\begin{array}{c}-9.4 \\
(-20.3,1.6)\end{array}$ \\
\hline
\end{tabular}

${ }^{*}$ Clinical responses defined as: Cure-the course of study drug and the initial intervention (operative and/or radiologically guided drainage procedure) resolved the intra-abdominal infectious process; Failure-the patient required additional antibacterial therapy other than the study drug, the patient required additional surgical or radiologic intervention to cure the infection, death due to infection occurred after 48 hours of therapy or a treatment-related adverse event (AE), or the patient received an extended course of study drug (i.e., > 120\% of the planned number of doses); and Indeterminate-the patient was lost to follow-up, or died within 48 hours after the first dose of study drug for any reason, or died after 48 hours because of noninfectious-related reasons (as judged by the investigator). 
Table 4 Clinical cure rates by diagnosis at test-of-cure visit (ME population)

\begin{tabular}{|c|c|c|c|c|c|}
\hline \multirow[b]{2}{*}{ Diagnosis } & \multicolumn{2}{|c|}{ Tigecycline } & \multicolumn{2}{|c|}{ Imipenem/cilastatin } & \multirow{2}{*}{$\begin{array}{c}\text { Difference (Tigecycline-Imipenem/cilastatin) } \\
\%(95 \% \mathrm{Cl})\end{array}$} \\
\hline & $\mathbf{N}$ & $\%(95 \% \mathrm{Cl})$ & $\mathbf{N}$ & $\%(95 \% \mathrm{Cl})$ & \\
\hline Complicated appendicitis & $40 / 46$ & $\begin{array}{c}87.0 \\
(73.7,95.1)\end{array}$ & $45 / 45$ & $\begin{array}{c}100 \\
(92.1,100.0)\end{array}$ & $\begin{array}{c}-13.0 \\
(-27.0,-0.6)\end{array}$ \\
\hline Complicated cholecystitis & $2 / 2$ & $\begin{array}{c}100.0 \\
(15.8,100.0)\end{array}$ & $0 / 0$ & NA & NA \\
\hline Peritonitis & $2 / 3$ & $\begin{array}{c}66.7 \\
(9.4,99.2)\end{array}$ & $2 / 3$ & $\begin{array}{c}66.7 \\
(9.4,99.2)\end{array}$ & $\begin{array}{c}0.0 \\
(-62.7,62.7)\end{array}$ \\
\hline Gastric/duodenal perforation & $1 / 1$ & $\begin{array}{c}100.0 \\
(2.5,100.0)\end{array}$ & $0 / 0$ & NA & NA \\
\hline
\end{tabular}

NA - not available

were mild to moderate in severity and not considered clinically important.

Nine SAEs were recorded during the study period (8 [8.2\%] tigecycline vs. $1[1.0 \%]$ imipenem/cilastatin) $(P=$ 0.016). The most frequently reported SAE was abnormal

(a) ME population

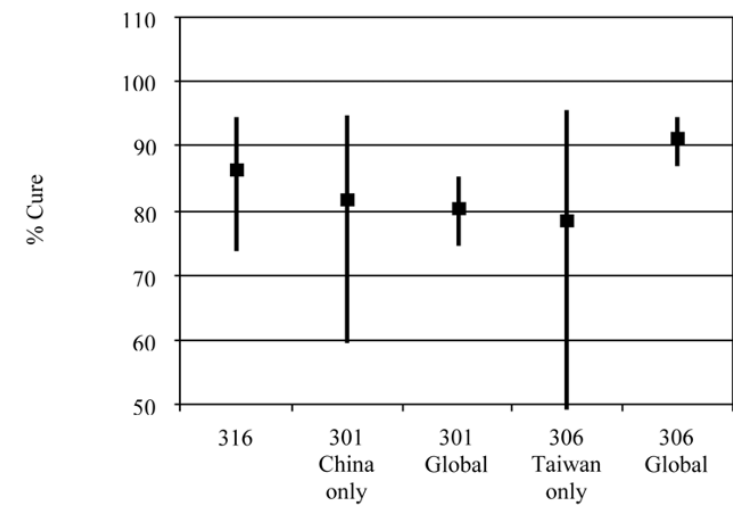

(b) m-mITT population

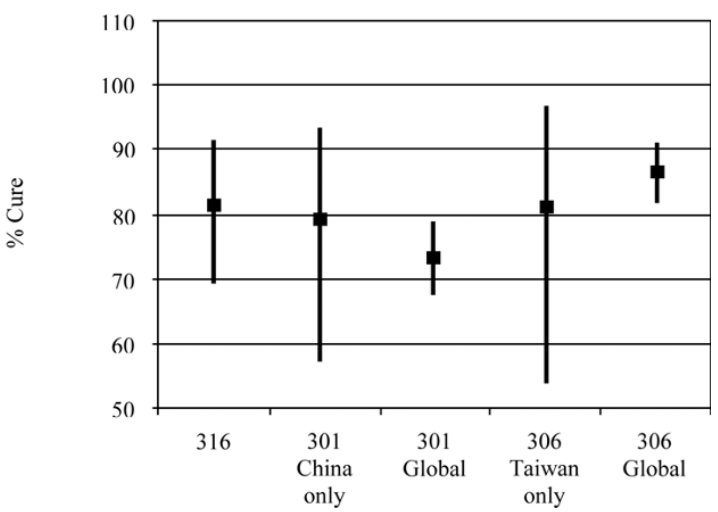

Figure 2 Tigecycline clinical cure rates compared to previous intra-abdominal studies $(301,306)$ : (a) microbiologically evaluable (ME) and (b) microbiologic modified intent-to-treat (m-mITT) populations. Error bars indicate $95 \%$ confidence intervals, unweighted and calculated using the method of Clopper and Pearson. healing (3 [3.1\%] tigecycline vs. 0 imipenem/cilastatin; $P=$ $0.114)$. There were no significant differences between the treatment groups in the specific types of SAEs experienced. Overall, these SAEs were not considered by the investigator to be related to study medication.

Two (2.1\%) tigecycline- and 2 (2.0\%) imipenem/cilastatin-treated patients had treatment stopped early because of an adverse event. In the tigecycline group, 1 patient discontinued therapy because of gastrointestinal hemorrhage and shock and 1 because of nausea. Both imipenem/cilastatin-treated patients discontinued treatment secondary to diarrhea.

A single death was reported during the study, which was unrelated to study medication. This tigecycline-treated patient, a 74-year-old man who underwent a colostomy, died of septic shock and multiple organ failure 1 day after his first dose of study medication.

The only clinically relevant laboratory test abnormality was that significantly more Chinese patients treated with tigecycline had a low platelet count (i.e., platelet count $\leq$ $\left.100 \times 10^{9} / \mathrm{L}\right)$ compared with the imipenem/cilastatintreated patients $(11.5 \%$ vs. $2.0 \%, P=0.009)$. Further examination of 7 patients who had platelet counts $\leq 50$ $\times 10^{9} / \mathrm{L}$ revealed that these patients either had thrombocytopenia at baseline, or thrombocytopenia was attributed by the investigator to infection/sepsis. Mean platelet count increases observed at the TOC assessment were similar in both treatment arms. There were no significant differences between the treatment groups with respect to mean changes in individual ECG parameters, nor were there any AEs associated with prolonged QT interval after either treatment reported.

\section{Discussion}

This analysis of hospitalized Chinese patients demonstrated that open-label tigecycline monotherapy (100 mg initial dose, followed by $50 \mathrm{mg}$ q12 hours) was effective for the treatment of cIAIs. The 199 patients included in the mITT population had mild to moderately severe intra-abdominal infections, as described by a mean APACHE II score of 4.6. For patients comprising the 
Table 5 Microbiologic response by patient at test-of-cure visit (ME population)

\begin{tabular}{|c|c|c|c|c|c|}
\hline & \multicolumn{2}{|c|}{ Tigecycline } & \multicolumn{2}{|c|}{ Imipenem/cilastatin } & \multirow{2}{*}{$\begin{array}{c}\begin{array}{c}\text { Difference } \\
\text { (Tigecycline-Imipenem/cilastatin) }\end{array} \\
\%(95 \% \mathrm{Cl})\end{array}$} \\
\hline & $\mathrm{N}$ & $\%(95 \% \mathrm{Cl})$ & $\mathrm{N}$ & $\%(95 \% \mathrm{Cl})$ & \\
\hline \multicolumn{6}{|l|}{ Overall } \\
\hline Eradication & $45 / 52$ & $\begin{array}{c}86.5 \\
(74.2,94.4)\end{array}$ & $47 / 48$ & $\begin{array}{c}97.9 \\
(88.9,99.9)\end{array}$ & $\begin{array}{c}-11.4 \\
(-23.5,0.7)\end{array}$ \\
\hline Persistence & $7 / 52$ & $\begin{array}{c}13.5 \\
(5.6,25.8)\end{array}$ & $1 / 48$ & $\begin{array}{c}2.1 \\
(0.1,11.1)\end{array}$ & \\
\hline Superinfection & $0 / 52$ & $\begin{array}{c}0 \\
(0.0,6.8)\end{array}$ & $0 / 48$ & $\begin{array}{c}0 \\
(0.0,7.4)\end{array}$ & \\
\hline \multicolumn{6}{|l|}{ Monomicrobial } \\
\hline Eradication & $30 / 33$ & $\begin{array}{c}90.9 \\
(75.7,98.1)\end{array}$ & $25 / 26$ & $\begin{array}{c}96.2 \\
(80.4,99.9)\end{array}$ & $\begin{array}{c}-5.2 \\
(-22.0,13.7)\end{array}$ \\
\hline Persistence & $3 / 33$ & $\begin{array}{c}9.1 \\
(1.9,24.3)\end{array}$ & $1 / 26$ & $\begin{array}{c}3.8 \\
(0.1,19.6)\end{array}$ & \\
\hline Superinfection & $0 / 33$ & $\begin{array}{c}0 \\
(0.0,10.6)\end{array}$ & $0 / 26$ & $\begin{array}{c}0 \\
(0.0,13.2)\end{array}$ & \\
\hline \multicolumn{6}{|l|}{ Polymicrobial } \\
\hline Eradication & 15/19 & $\begin{array}{c}78.9 \\
(54.4,93.9)\end{array}$ & $22 / 22$ & $\begin{array}{c}100.0 \\
(84.6,100.0)\end{array}$ & $\begin{array}{c}-21.1 \\
(-46.1,2.2)\end{array}$ \\
\hline Persistence & $4 / 19$ & $\begin{array}{c}21.1 \\
(6.1,45.6)\end{array}$ & $0 / 22$ & $\begin{array}{c}0 \\
(0.0,15.4)\end{array}$ & \\
\hline Superinfection & 0/19 & $\begin{array}{c}0 \\
(0.0,17.6)\end{array}$ & $0 / 22$ & $\begin{array}{c}0 \\
(0.0,15.4)\end{array}$ & \\
\hline
\end{tabular}

ME and m-mITT populations, clinical cure rates ranged from $81.7 \%$ to $86.5 \%$ for tigecycline vs. $90.9 \%$ to $97.9 \%$ for imipenem/cilastatin at the test-of-cure visit. All ME patients with bacteremia in both treatment groups were clinically cured; however, the absolute number of such patients was small. Tigecycline-treated patients with polymicrobial infection tended to have lower clinical cure rates compared with patients who had monomicrobial infection. Although approximately three-quarters of patients had complicated appendicitis, tigecycline was effective across the range of clinical diagnoses.
Microbiologic responses paralleled clinical outcomes for both tigecycline and imipenem/cilastatin in Chinese patients. In the ME population, the baseline organisms were eradicated in $86.5 \%$ of tigecycline-treated patients and $97.9 \%$ of patients treated with imipenem/cilastatin at the TOC visit. Satisfactory eradication of commonly encountered aerobic and anaerobic intestinal bacteria after both treatments was also observed. Eradication rates for $E$. coli, the most commonly isolated bacteria, were $88.1 \%$ for tigecycline vs. $97.7 \%$ for imipenem/cilastatin. Although other Gram-negative enterics (e.g.,

Table 6 Microbiologic eradication at the isolate level: selected baseline isolates at test-of-cure visit (ME population)

\begin{tabular}{|c|c|c|c|c|c|c|}
\hline \multirow[b]{2}{*}{ Baseline Isolate } & \multicolumn{3}{|c|}{ Tigecycline $(\mathrm{N}=52)$} & \multicolumn{3}{|c|}{ Imipenem/cilastatin $(\mathrm{N}=48)$} \\
\hline & $\mathrm{N}$ & $E$ & ER (\%) & $\mathrm{N}$ & $\mathrm{E}$ & ER (\%) \\
\hline Total & 83 & 69 & 83.1 & 75 & 74 & 98.7 \\
\hline Gram negative aerobic bacteria & 56 & 48 & 85.7 & 59 & 58 & 98.3 \\
\hline E. coli & 42 & 37 & 88.1 & 44 & 43 & 97.7 \\
\hline Other Enterobacteriaceae & 8 & 6 & 75.0 & 9 & 9 & 100 \\
\hline Non-fermentative Gram-negative bacilli & 6 & 5 & 83.3 & 6 & 6 & 100 \\
\hline Gram positive aerobic bacteria & 15 & 12 & 80.0 & 8 & 8 & 100 \\
\hline Enterococcus spp. & 10 & 8 & 80.0 & 5 & 5 & 100 \\
\hline Streptococcus spp. & 5 & 4 & 80.0 & 2 & 2 & 100 \\
\hline Staphylococcus spp. & 0 & 0 & 0 & 1 & 1 & 100 \\
\hline Anaerobe & 12 & 9 & 75.0 & 8 & 8 & 100 \\
\hline Bacterioides spp. & 7 & 5 & 71.4 & 6 & 6 & 100 \\
\hline Other anaerobe & 5 & 4 & 80.0 & 2 & 2 & 100 \\
\hline
\end{tabular}

$\mathrm{E}=$ eradication (presumed), and $\mathrm{ER}=$ eradication rate. 
Table 7 Common treatment-emergent adverse events ( $\geq 3 \%$ in either group)

\begin{tabular}{|c|c|c|c|}
\hline $\begin{array}{l}\text { Body System } \\
\text { Adverse Event }\end{array}$ & Tigecycline $(\mathrm{N}=97)$ & Imipenem/cilastatin $(\mathrm{N}=102)$ & $P$ value $^{a}$ \\
\hline Any adverse event & $78(80.4)$ & $55(53.9)$ & $<0.001$ \\
\hline Body as a whole & $11(11.3)$ & $8(7.8)$ & 0.473 \\
\hline Chest pain & $3(3.1)$ & $1(1.0)$ & 0.359 \\
\hline Digestive system & $35(36.1)$ & $16(15.7)$ & 0.001 \\
\hline Abdominal distension & $7(7.2)$ & $2(2.0)$ & 0.094 \\
\hline Diarrhea & $5(5.2)$ & $9(8.8)$ & 0.409 \\
\hline Nausea & $21(21.6)$ & $4(3.9)$ & $<0.001$ \\
\hline Vomiting & $12(12.4)$ & $2(2.0)$ & 0.005 \\
\hline Hemic and lymphatic system & $23(23.7)$ & $21(20.6)$ & 0.613 \\
\hline Anemia & $3(3.1)$ & $3(2.9)$ & 1.000 \\
\hline Coagulation disorder & $4(4.1)$ & $0(0)$ & 0.055 \\
\hline Monocytosis & $1(1.0)$ & $6(5.9)$ & 0.119 \\
\hline Prothrombin time prolonged & $1(1.0)$ & $4(3.9)$ & 0.369 \\
\hline Thrombocythemia & $6(6.2)$ & $6(5.9)$ & 1.000 \\
\hline Thrombocytopenia & $5(5.2)$ & $1(1.0)$ & 0.111 \\
\hline Metabolic and nutritional & $37(38.1)$ & $35(34.3)$ & 0.658 \\
\hline ALT/SGPT increased & $3(3.1)$ & $5(4.9)$ & 0.722 \\
\hline AST/SGOT increased & $6(6.2)$ & $7(6.9)$ & 1.000 \\
\hline Amylase increased & $8(8.2)$ & $9(8.8)$ & 1.000 \\
\hline Bilirubinemia & 21 (21.6) & $12(11.8)$ & 0.085 \\
\hline Healing abnormal & $7(7.2)$ & $6(5.9)$ & 0.779 \\
\hline Hyperglycemia & $1(1.0)$ & $4(3.9)$ & 0.369 \\
\hline Hypocalcemia & $6(6.2)$ & $5(4.9)$ & 0.763 \\
\hline Hypokalemia & $1(1)$. & $4(3.9)$ & 0.369 \\
\hline Hyponatremia & $3(3.1)$ & $1(1.0)$ & 0.359 \\
\hline Hypophosphatemia & $5(5.2)$ & $5(4.9)$ & 1.000 \\
\hline Hypoproteinemia & $10(10.3)$ & $11(10.8)$ & 1.000 \\
\hline Lipase increased & $3(3.1)$ & $0(0)$ & 0.114 \\
\hline Respiratory system & $3(3.1)$ & $3(2.9)$ & 1.000 \\
\hline Cough increased & $3(3.1)$ & $1(1.0)$ & 0.359 \\
\hline Skin and appendages & $1(1.0)$ & $4(3.9)$ & 0.369 \\
\hline Rash & $1(1.0)$ & $4(3.9)$ & 0.369 \\
\hline Adverse event associated with miscellaneous factors & $4(4.1)$ & $6(5.9)$ & 0.748 \\
\hline Local reaction to procedure & $4(4.1)$ & $6(5.9)$ & 0.748 \\
\hline
\end{tabular}

AST/SGOT = aspartate aminotransferase/serum glutamic oxaloacetic transaminase. ALT/SGOT = alanine aminotransferase/serum glutamic oxaloacetic transaminase.

a Between-group comparisons of adverse events were analyzed by using the Fisher exact test. Significant between-group difference at $P \leq 0.05$ level.

K. pneumoniae, P. mirabilis), Gram-positive (e.g., Streptococcus spp., Enterococcus spp.), and anaerobic bacteria (e.g., B. fragilis, Bacteroides spp.) were isolated in small numbers, tigecycline generally eradicated most of these pathogens. Overall, these data provide in vivo evidence that tigecycline has broad-spectrum activity against common bacterial etiologies associated with cIAIs $[9,10,14,17,18]$.

Tigecycline monotherapy was generally well tolerated in this study population, even though the overall incidence of treatment-emergent AEs was significantly higher after tigecycline therapy (80.4\%) compared with imipenem/cilastatin $(53.9 \% ; P<0.001)$. The main reason for the increased rates of AEs was gastrointestinal, with tigecycline-treated patients having a two-fold higher rate than those given imipenem/cilastatin $(P=0.001)$. In particular, nausea and vomiting occurred significantly more often (5-6 fold) after tigecycline therapy. To the contrary, diarrhea occurred in higher rates among imipenem/cilastatin recipients $(P>0.05)$. Gastrointestinalrelated adverse events rarely led to early discontinuation of either therapy, however, as only one tigecycline patient stopped treatment early due to nausea, as did two imipenem/cilastatin-treated patients secondary to diarrhea. Of interest, nearly two-fold more patients treated with tigecycline had bilirubinemia compared with 
patients treated with imipenem/cilastatin $(21.6 \%$ vs. $11.8 \% ; P=0.085$ ). While bilirubinemia has been observed in prior studies of tigecycline to treat cIAI, the rates in the current study were higher than those observed in patients enrolled in previous trials (16); in each instance, however, the presence of mitigating factors confound determinations of a relationship between the study drugs and changes in bilirubin levels. Overall, safety/tolerability findings described herein were consistent with the original global trials [16] and support previous safety data from phase 2 and 3 studies [16,19-24]. Significantly more subjects in the tigecycline treatment arm had SAEs than subjects in the imipenem/cilastatin $\operatorname{arm}(8.2 \%$ vs. $1.0 \%, P=0.016)$; however, there were no significant differences between the treatment groups in the specific type of SAE reported. The most frequently reported SAE was abnormal healing. No unusual or novel adverse events were reported in Chinese patients after tigecycline monotherapy.

A major limitation of the current trial is that it was not powered for a formal statistical analysis of noninferiority. In addition, the study design was open-label (unblinded), adding to the confounding factors affecting comparisons of efficacy within the study. Accordingly, it is inappropriate to draw conclusions about the efficacy of tigecycline on the basis of the results of this study alone. However, the results of our study in Chinese patients is consistent with the efficacy findings from 2 global randomized, double-blind studies [16], and the same dose administration schedule was used across the 3 studies to treat hospitalized patients with cIAI. In study $301,80.6 \%$ of tigecycline-treated patients and $82.4 \%$ of imipenem/cilastatintreated patients in the ME population were clinically cured, as were $73.5 \%$ of tigecycline-treated patients and $78.2 \%$ of imipenem/cilastatin-treated patients in the mmITT population. In the overall ME population of study $306,91.3 \%$ of tigecycline-treated patients and $89.9 \%$ of imipenem/cilastatin-treated patients were clinically cured; in the m-mITT population, $86.6 \%$ of tigecyclinetreated subjects and $84.6 \%$ of imipenem/cilastatin-treated subjects were clinically cured. Furthermore, the 2 global studies included sites that enrolled Chinese patients [16]. Formal statistical analyses in the global studies demonstrated that tigecycline met the statistical criteria of noninferiority to imipenem/cilastatin for the primary endpoint of clinical response (cure or failure) in the co-primary populations at the TOC assessment, and the results of primary clinical cure or failure analyses for tigecycline in the study described herein mirrored the results in these pivotal cIAI studies.

\section{Conclusion}

In summary, tigecycline monotherapy appears to be both effective and safe for the treatment of cIAI in
Chinese patients. Both the efficacy and safety analyses for this study are consistent with the profile of tigecycline elucidated in the pivotal trials (Study 301/306) [16]. Digestive-related AEs were significantly higher in the tigecycline group, especially nausea and vomiting. With the diverse bacteriology of cIAIs and the emergence of bacterial resistance, tigecycline provides an empiric monotherapy option with coverage against a broad range of Gram-positive and Gram-negative aerobic and anaerobic bacteria, including resistant isolates based upon in vitro data.

\section{Acknowledgements}

These results were presented, in part, at the $10^{\text {th }}$ Western Pacific Congress on Chemotherapy and Infectious Diseases, Taipei, Taiwan, 2008, Abstract AP007.

\section{Author details}

${ }^{1}$ Huashan Hospital, Fudan University, Shanghai, China. ${ }^{2}$ Beijing Hospital, Beijing, China. ${ }^{3}$ Peking University People's Hospital, Beijing, China. ${ }^{4}$ Shanghai Changhai Hospital, Shanghai, China. ${ }^{5}$ China PLA General Hospital, Beijing, China. ${ }^{6}$ West China Hospital, Sichuan University, Chengdu, China. ${ }^{7}$ Shanghai Xinhua Hospital, Shanghai, China. ${ }^{8}$ Tongji Hospital, Wuhan, China. ${ }^{9}$ First Affiliated Hospital, College of Medicine, Zhejiang University, Hangzhou, Zhejiang, China. ${ }^{10}$ Shanghai Renji Hospital, Shanghai, China. ${ }^{11}$ Peking Union Medical College Hospital, Beijing, China. ${ }^{12}$ No. 1 Affiliated Hospital, China Medical University, Shengyang, LiaoNing, China. ${ }^{13}$ Pfizer Inc., Collegeville, PA, USA

\section{Authors' contributions}

$Z C$, J Wu, YZ, J Wei, $X L, J B, R L, L Y, Z Q, X C, Y Y, X W, D L$, and $X M$ conducted the study, contributed to data acquisition, and reviewed and approved the draft manuscript. RM and AC contributed to, reviewed, and approved the draft manuscript. All authors reviewed and approved the final version.

\section{Competing interests}

This study and analysis was sponsored by Wyeth Research, Collegeville, PA, USA, which was acquired by Pfizer, Inc. in October 2009. Pfizer assumes responsibility for the study design, and collection, analysis, and interpretation of the data. Pfizer provided the authors with editorial support for the preparation of this publication (Upside Endeavors, LLC, Sanatoga, PA, USA). Zhangjing Chen, Robert Maroko, and Angel Cooper are employees of Pfizer, Inc. Zhangjing Chen was one of the investigators in studies 316 and 301. Jufang Wu, Yingyuan Zhang, Junming Wei, Xisheng Leng, Jianwei Bi, Rong Li, Lunan Yan, Zhiwei Quan, Xiaoping Chen, Yunsong Yu, Zhiyong Wu, Dawei Liu, and Xiaochun Ma have no competing interests to declare.

Received: 22 May 2009 Accepted: 21 July 2010 Published: 21 July 2010

\section{References}

1. Mazuski JE, Sawyer RG, Nathens AB, DiPiro JT, Schein M, Kudsk KA Yowler C: The Surgical Infection Society guidelines on antimicrobial therapy for intra-abdominal infections: evidence for the recommendations. Surg Infect (Larchmt) 2002, 3(3):175-233.

2. Solomkin JS, Mazuski JE, Baron EJ, Sawyer RG, Nathens AB, DiPiro JT, Buchman T, Dellinger EP, Jernigan J, Gorbach S, et al: Guidelines for the selection of anti-infective agents for complicated intra-abdominal infections. Clin Infect Dis 2003, 37(8):997-1005.

3. Weigelt JA: Empiric treatment options in the management of complicated intra-abdominal infections. Cleve Clin J Med 2007, 74(Suppl 4):S29-37.

4. Nathens $A B$, Rotstein $O D$, Marshall JC: Tertiary peritonitis: clinical features of a complex nosocomial infection. World J Surg 1998, 22(2):158-163.

5. Krobot K, Yin D, Zhang Q, Sen S, Altendorf-Hofmann A, Scheele J, Sendt W: Effect of inappropriate initial empiric antibiotic therapy on outcome of 
patients with community-acquired intra-abdominal infections requiring surgery. Eur J Clin Microbiol Infect Dis 2004, 23(9):682-687.

6. Marshall $\mathrm{JC}$, Innes $\mathrm{M}$ : Intensive care unit management of intra-abdominal infection. Crit Care Med 2003, 31(8):2228-2237.

7. Stein GE, Craig WA: Tigecycline: a critical analysis. Clin Infect Dis 2006, 43(4):518-524.

8. Chopra I: New developments in tetracycline antibiotics: glycylcyclines and tetracycline efflux pump inhibitors. Drug Resist Updat 2002, 5(34):119-125.

9. Gales AC, Sader HS, Fritsche TR: Tigecycline activity tested against 11808 bacterial pathogens recently collected from US medical centers. Diagn Microbiol Infect Dis 2008, 60(4):421-427.

10. Hoban DJ, Bouchillon SK, Johnson BM, Johnson JL, Dowzicky MJ: In vitro activity of tigecycline against 6792 Gram-negative and Gram-positive clinical isolates from the global Tigecycline Evaluation and Surveillance Trial (TEST Program, 2004). Diagn Microbiol Infect Dis 2005, 52(3):215-227.

11. Zhanel GG, Karlowsky JA, Rubinstein E, Hoban DJ: Tigecycline: a novel glycylcycline antibiotic. Expert Rev Anti Infect Ther 2006, 4(1):9-25.

12. Castanheira M, Sader HS, Deshpande LM, Fritsche TR, Jones RN: Antimicrobial activities of tigecycline and other broad-spectrum antimicrobials tested against serine carbapenemase- and metallo-betalactamase-producing Enterobacteriaceae: report from the SENTRY Antimicrobial Surveillance Program. Antimicrob Agents Chemother 2008, 52(2):570-573.

13. Draghi DC, Tench S, Dowzicky MJ, Sahm DF: Baseline in vitro activity of tigecycline among key bacterial pathogens exhibiting multidrug resistance. Chemotherapy 2008, 54(2):91-100.

14. Fritsche TR, Strabala PA, Sader HS, Dowzicky MJ, Jones RN: Activity of tigecycline tested against a global collection of Enterobacteriaceae, including tetracycline-resistant isolates. Diagn Microbiol Infect Dis 2005, 52(3):209-213.

15. Meagher AK, Ambrose PG, Grasela TH, Ellis-Grosse EJ: The pharmacokinetic and pharmacodynamic profile of tigecycline. Clin Infect Dis 2005, 41(Suppl 5):S333-340.

16. Babinchak T, Ellis-Grosse E, Dartois N, Rose GM, Loh E: The efficacy and safety of tigecycline for the treatment of complicated intra-abdominal infections: analysis of pooled clinical trial data. Clin Infect Dis 2005, 41(Suppl 5):S354-367.

17. Gales AC, Jones RN, Andrade SS, Pereira AS, Sader HS: In vitro activity of tigecycline, a new glycylcycline, tested against 1,326 clinical bacterial strains isolated from Latin America. Braz J Infect Dis 2005, 9(5):348-356.

18. Sader HS, Jones RN, Dowzicky MJ, Fritsche TR: Antimicrobial activity of tigecycline tested against nosocomial bacterial pathogens from patients hospitalized in the intensive care unit. Diagn Microbiol Infect Dis 2005, 52(3):203-208.

19. Tanaseanu C, Bergallo C, Teglia O, Jasovich A, Oliva ME, Dukart G, Dartois N, Cooper CA, Gandjini H, Mallick R: Integrated results of 2 phase 3 studies comparing tigecycline and levofloxacin in community-acquired pneumonia. Diagn Microbiol Infect Dis 2008, 61(3):329-338.

20. Fomin P, Beuran M, Gradauskas A, Barauskas G, Datsenko A, Dartois N, EllisGrosse E, Loh E, Three Hundred Six Study G: Tigecycline is efficacious in the treatment of complicated intra-abdominal infections. Int J Surg 2005, 3(1):35-47.

21. Breedt J, Teras J, Gardovskis J, Maritz FJ, Vaasna T, Ross DP, GioudPaquet M, Dartois N, Ellis-Grosse EJ, Loh E: Safety and efficacy of tigecycline in treatment of skin and skin structure infections: results of a double-blind phase 3 comparison study with vancomycin-aztreonam. Antimicrob Agents Chemother 2005, 49(11):4658-4666.

22. Sacchidanand S, Penn RL, Embil JM, Campos ME, Curcio D, Ellis-Grosse E, Loh E, Rose G: Efficacy and safety of tigecycline monotherapy compared with vancomycin plus aztreonam in patients with complicated skin and skin structure infections: Results from a phase 3, randomized, doubleblind trial. Int J Infect Dis 2005, 9(5):251-261.

23. Ellis-Grosse EJ, Babinchak T, Dartois N, Rose G, Loh E: The efficacy and safety of tigecycline in the treatment of skin and skin-structure infections: results of 2 double-blind phase 3 comparison studies with vancomycin-aztreonam. Clin Infect Dis 2005, 41(Suppl 5):S341-353.

24. Muralidharan G, Micalizzi M, Speth J, Raible D, Troy S: Pharmacokinetics of tigecycline after single and multiple doses in healthy subjects. Antimicrob Agents Chemother 2005, 49(1):220-229.

\section{Pre-publication history}

The pre-publication history for this paper can be accessed here: http://www.biomedcentral.com/1471-2334/10/217/prepub

doi:10.1186/1471-2334-10-217

Cite this article as: Chen et al:: Efficacy and safety of tigecycline monotherapy vs. imipenem/cilastatin in Chinese patients with complicated intra-abdominal infections: a randomized controlled trial. BMC Infectious Diseases 2010 10:217.

\section{Submit your next manuscript to BioMed Central and take full advantage of:}

- Convenient online submission

- Thorough peer review

- No space constraints or color figure charges

- Immediate publication on acceptance

- Inclusion in PubMed, CAS, Scopus and Google Scholar

- Research which is freely available for redistribution

Submit your manuscript at www.biomedcentral.com/submit
Ciomed Central 